\title{
Paper
}

\section{Fast and Accurate Whole-Body Pose Estimation in the Wild and Its Applications}

\author{
Jianfeng XU (member) ${ }^{\dagger}$, Kazuyuki TASAKA (member) ${ }^{\dagger}$, Masashi YAMAGUCHI ${ }^{\dagger \dagger}$
}

\begin{abstract}
Recently, multi-person pose estimation techniques have drawn significant attention in both academia and industry due to their great potential utility. Here, we present a fast and accurate in-the-wild whole-body pose estimation system. Our system detects not only body keypoints, but also foot and hand keypoints, with high accuracy in real time. Furthermore, we present two typical applications of our system, in the areas of fitness and speed climbing, and describe the optimization of these applications, which has yielded both computing acceleration and improved detection accuracy.
\end{abstract}

Key words: whole-body pose estimation, applications, fitness, speed climbing, in the wild.

\section{Introduction}

Human pose estimation based on monocular images aims to localize human joints (also known as keypoints, see Fig. 1) in images, and is widely used in humancomputer interactions, gaming, virtual reality, video surveillance, sports analysis, and medical assistance ${ }^{1)}$. In the last decade, many new techniques have been proposed for human pose estimation with the development of convolutional neural networks, making it a highly popular research topic in the field of computer vision ${ }^{2}$. In this paper, we focused on multi-person pose estimation, which has no limitation on the number of individuals in the images.

The popular multi-person pose estimation techniques can be categorized into the bottom-up and top-down approaches ${ }^{2)}$. The bottom-up approaches such as OpenPose $^{3)}$ first detect the heatmap of keypoints in all persons from the images, then connect the body joints or keypoints for each person. One advantage of bottom-up approaches is that there is little change in the computational cost regardless of the number of persons $^{3)}$. The top-down approaches such as $\mathrm{CPN}^{4)}$, AlphaPose $^{5)}$, and HRNet ${ }^{6)}$ first detect each person's area with a bounding box, using, for example, faster $\mathrm{R}-\mathrm{CNN}^{7)}$ in AlphaPose, then estimate the keypoints in

\footnotetext{
Received August 19, 2020; Revised November 23, 2020; Accepted December 16, 2020

$\dagger$ KDDI Research, Inc.

(Saitama, Japan)

$\dagger \dagger$ KDDI Corporation

(Tokyo, Japan)

This paper includes videos. Note that the videos are not viewable from this PDF file. The videos are available as separate files on the website that hosts this PDF file.
}

each detected person area. Generally speaking, the accuracy of top-down approaches is better than that of bottom-up approaches ${ }^{8)}$, so, we adopt the top-down approach in this paper.

Object detection is the first step in the top-down approaches of multi-person pose estimation techniques, where many techniques ${ }^{9)}$ are available as briefly surveyed in Section 2. After human detection, a deep convolutional neural network is designed to obtain heatmaps of keypoints from each person area $^{3)^{\sim 610)}}$. Because the pixel value in a heatmap indicates the keypoint existence probability at that position, the maximal value in a heatmap determines the confidence of the keypoint, whose position in the heatmap is the estimated position of the keypoint. Therefore, for the $k$-th keypoint in the $i$-th person, we can get a $2 \mathrm{D}$ position $\boldsymbol{P}(i, k)$ and its confidence level $f(i, k)$.

Although the state-of-the-art technique of multiperson pose estimation is powerful, there are many practical issues in a real environment ${ }^{11)}$. In this paper, we will report three of them and propose our solutions. First, some applications may require special keypoints. For example, the foot joints are useful in soccer analysis. Second, some applications require real time processing and allow simultaneous use from many users. Our solution is given in Sect. 4. Third, in the real environment, the accuracy of keypoints may decline due to factors such as shadows in speed climbing, see Sect. 5 .

The remainder of this paper is organized as follows. After a brief survey in Sect. 2, we will introduce our system in Sect. 3. Then, we describe two applications in detail in Sect. 4 and Sect. 5, where we discuss some technical issues in these real environments. In Sect. 6, 


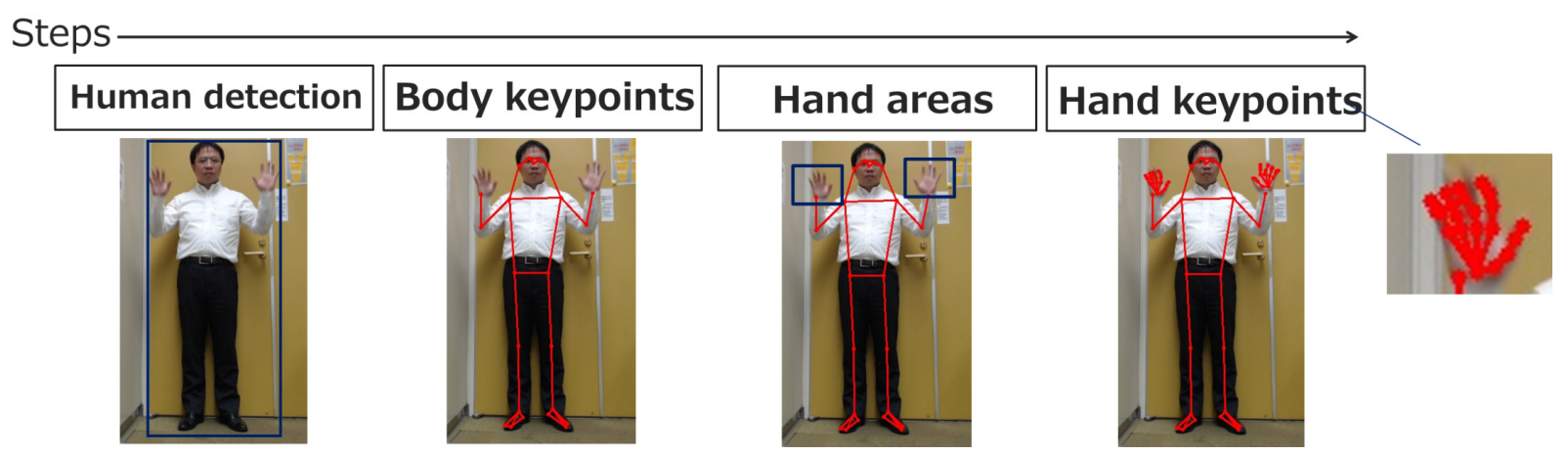

Fig. 1 Pipeline of our system: object detection, body keypoint detection with foot joints, hand area estimation, and hand keypoint detection.

we will discuss the technical issues in multi-person applications such as soccer analysis. Finally, we present our conclusions in Sect. 7.

\section{Related Works}

In this section, we briefly survey techniques related to our approach, i.e., object detection and pose estimation. There are survey papers that fully cover the object detection field ${ }^{9)}$ and the pose estimation field ${ }^{12)}$.

\subsection{Object detection}

As a state-of-the-art technique in object detection, faster R-CNN ${ }^{7)}$ used a two-stage object detection strategy. It achieved very high detection accuracy within the most commonly used datasets such as COCO. However, the computational cost was still high, basically requiring faster R-CNN to run in GPU mode. Therefore, single shot detectors such as the YOLO family of object detectors ${ }^{13)^{-15)}}$ and $\mathrm{SSD}^{16)}$ were proposed, which ran much faster with reasonably high accuracy ${ }^{17)}$. $\mathrm{YOLO}^{13)}$ was an end-to-end single convolutional neural network that detected objects based on bounding boxes prediction and class probabilities. However, it was still difficult to detect small-sized objects and achieve precise localization. Thereafter, SSD ${ }^{16)}$ was proposed for improving the YOLO-based method. With the introduction of multiscale feature maps and the default boxes mechanism, SSD ${ }^{16)}$ can detect small-sized objects and also improve localization accuracy compared with $\mathrm{YOLO}^{13)}$. On the other hand, YOLO has evolved and solved its initial problem. The latest version, YOLOv ${ }^{15)}$, carries out detection on three different scales and utilizes a more powerful deep architecture that has more layers with residual blocks.

\subsection{Pose estimation}

In the past decade, many papers on multi-person pose estimation have been published ${ }^{12)}$. They are usually divided into two categories: bottom-up and top-down approaches. As a typical bottom-up approach, OpenPose $^{18)}$ detected all the body joints in the first stage, then associated them with person instances in the second stage. OpenPose ${ }^{18)}$ used a non-parametric representation called Part Affinity Fields (PAFs) with a greedy algorithm to generate the person instance.

On the other hand, top-down approaches reported better performance, where they first detected the bounding boxes of persons in the input image, then estimated the joint locations in each bounding box. Fang et al.5) noticed that single-person pose estimation is sensitive to human detection. To solve this problem, they employed Symmetric Spatial Transformer Network (SSTN) in parallel with Single-Person Pose Estimator (SPPE) to extract a high-quality single-person region. Mask R-CNN ${ }^{19)}$ simultaneously predicted bounding boxes and body joints, which made the detection faster by sharing the features. Moreover, the new RoI alignment method enabled more accurate feature cropping. Chen et al. ${ }^{4}$ proposed a network structure called Cascaded Pyramid Network (CPN), which consisted of two parts, GlobalNet and RefineNet. The former extracted a good feature representation, while the latter was employed to address the "hard" examples. The latest technology of $\mathrm{HRNet}^{20)}$ proposed an architecture that preserves high-resolution feature maps, which has been shown to be highly beneficial in multi-person pose estimation tasks. HRNet ${ }^{20)}$ consisted of multiple branches with different resolutions. Lower resolution branches captured contextual information and higher resolution branches preserved spatial information. With multi-scale fusions between branches, HR$\mathrm{Net}^{20)}$ can generate high resolution feature maps with rich semantic content. Recently, a new dataset with whole-body annotations ${ }^{10)}$ was publicly released, which 
extends COCO dataset. Moreover, a single-network model $^{10)}$ was proposed for whole-body pose estimation, where previous methods used assemble deep models trained independently on different datasets of the human body and hands.

Note that in all the papers mentioned above a heatmap is generated for each joint, in which the pixel value indicates the joint existence probability at that location. Zhang et al. $^{21)}$ regarded heatmap as the de facto standard coordinate representation in human pose estimation.

\section{Framework}

As mentioned in Sect. 1, we adopt a top-down approach in this paper due to its high accuracy. Moreover, we aim to obtain even better accuracy, faster computing, and more keypoints that can work well in the wild. Fig. 1 shows the pipeline of our system. In the first step, we detect persons by using an object detector. In the applications introduced later, single shot detectors such as $\mathrm{SSD}^{16)}$ or YOLO v3 ${ }^{15)}$ are shown to provide a good balance between computational cost and accuracy.

In the second step, we adopt a similar network as $\mathrm{CPN}^{4)}$ for body keypoints * However, one problem is that the original $\mathrm{CPN}^{4}$ cannot detect the six footjoints. Therefore, we extended it by transfer learning with CMU's foot dataset as follows.

- Extend the keypoint number to cover the foot joints in the network structure. Then, train the new network using the original model as the pre-trained model, where we set the positions of all six foot joints as zeros in the COCO training data ${ }^{22)}$.

- Fine-tuning the above model with CMU's foot dataset (https://cmu-perceptual-computing-lab. github.io/foot_keypoint_dataset/ $)^{23)}$, which is much smaller than the COCO dataset ${ }^{22)}$. In this phase, only the parameters for the six foot joints are trained.

In the third step, we infer the hands' positions and their areas based on the wrist joints, elbow joints, and shoulder joints. We assume that a hand area is a square with length $L$ and its center at $H$. As shown in Fig. 2, we calculate the length and position of the hand area using Eq. (1) and Eq. (2).

$$
\begin{array}{r}
\|W H\|=\text { ratio } 1 *\|E W\| \\
L=\text { ratio } 2 * \max (\|E W\|, \text { ratio } 3 *\|S E\|)
\end{array}
$$

where $W, E, S$, and $H$ are the positions of wrist joints,

\footnotetext{
* Note that you can select any other technique ${ }^{6}$ you wish
}

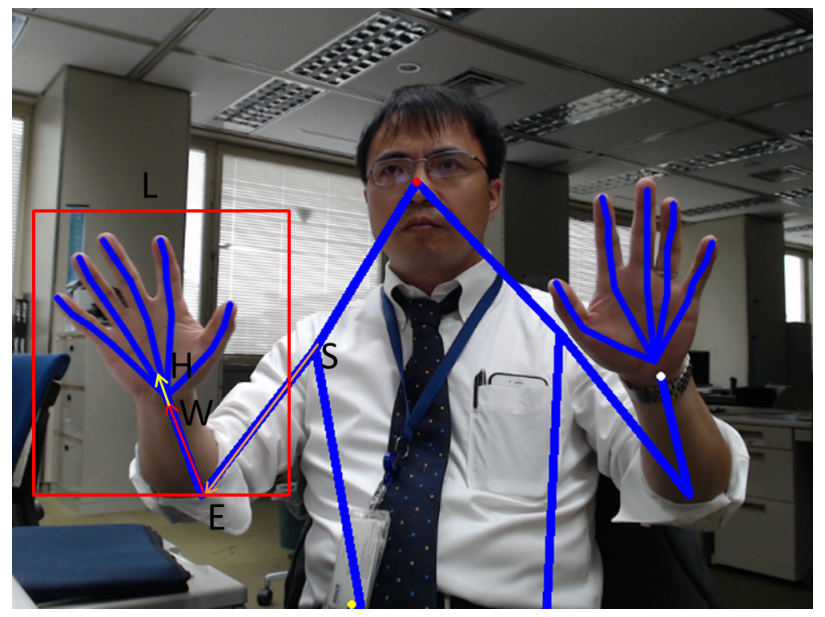

Fig. 2 Hand area estimation using the wrist joint $W$, elbow joint $E$, and shoulder joint $S$. Only right hand is shown.

elbow joints, shoulder joints, and hand area centers, $L$ denotes the length of a hand area, $\|X Y\|$ denotes the Euclidean distance between two joints of $X$ and $Y$, ratio1, ratio 2 , and ratio 3 are three ratios, which are determined empirically. In our implementation, ratio1, ratio 2 , and ratio 3 are set as $0.33,1.5$, and 0.9 , respectively.

In the last step, the hand keypoints can be detected using an independent model that has been trained on CMU's hand dataset (http://domedb. perception. cs.cmu.edu/handdb.html) $)^{24)}$. Because there are two hands for each person, batch processing is recommended. In our server, it was demonstrated that batch processing almost halved computing time with no loss of accuracy in hand pose estimation.

Although a single-network model for whole-body pose estimation was proposed in ECCV 202010), it requires deeper and complex networks due to the challenging scale problem (i.e., the areas of hand and body have very different scales in images). In addition, the proposed method is more flexible for different applications because it uses ensemble models. For example, in some applications like playing piano, we need to load the hand model. But in other applications like running, we do not load the hand model, which reduces the computing cost much.

\section{Application to Fitness}

The first application we introduce in this paper is fitness. Traditionally, broadcasters like NHK provide fitness programs at home. Recently, many applications in smart-phones provide similar services. However, users of these applications cannot get any feedback on the de- 


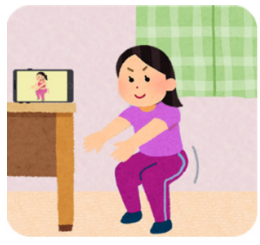

Home

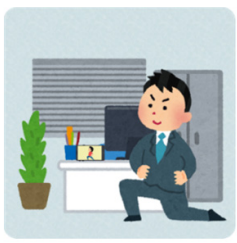

Office

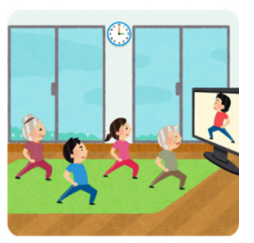

Public hall
Fig. 3 Application to Fitness. Our service is available using only a smart-phone at different places. From left to right: home, office, and public hall.

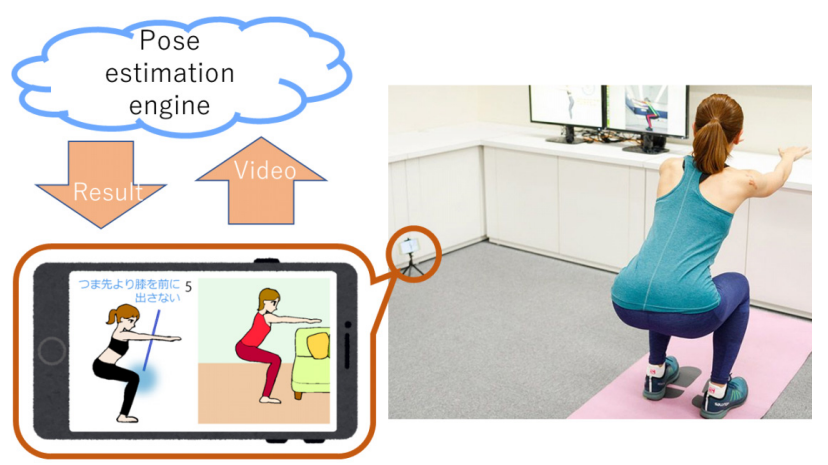

Fig. 4 System of Fitness Application. The application in a smart-phone captures the user's video and sends it to our server, then receives the poses from the server and gives the user real-time advice.

picted movement. The unique service our application provides is that the users will receive useful advice in real time. For example, the users will know whether to bend their legs more or less, depending on the knee's angle.

As shown in Fig. 3, if the user just installs our fitness application in his/her smart-phone, our service will be available anytime and anywhere: from home, office or public hall. From the smart-phone, the application sends the videos to our server, and the poses in the video will be estimated in the server in real time and sent back to the application as shown in Fig. 4. According to the poses, our application will give the user some advice in real time, which is designed for each menu. Fig. 5 shows some menu samples in our application.

In the fitness application, we have improved the accuracy of pose estimation and accelerated the computing in the server. The poses in some menus of fitness are very challenging even when using the state-of-the-art technique, as they are rare and far away from the training data in the $\mathrm{COCO}$ dataset ${ }^{22)}$ as shown in Fig. 7 . By creating a particular dataset for fitness, we fine-tune the model and get much better performance as shown in Fig. 6. We collect one hundred images for an easy menu in our application while we collect two hundred

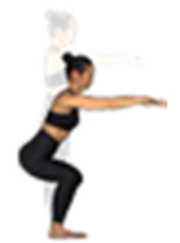

squat

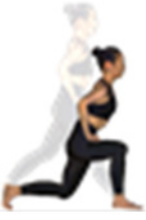

lunge

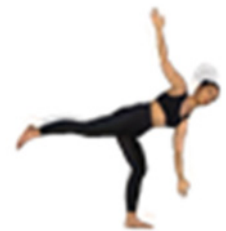

propel

Fig. 5 Menu samples of Fitness Application. For each menu, our application can generate useful and specific feedback according to the pose estimation.

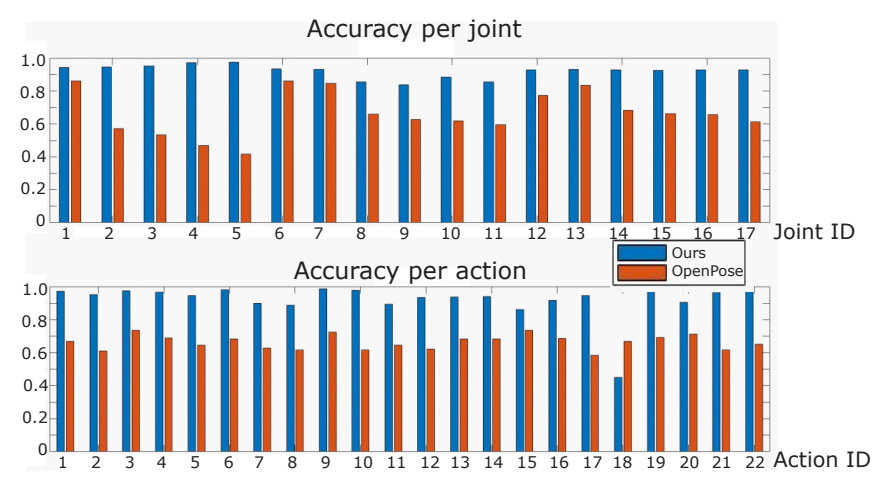

Fig. 6 Our system achieves much higher accuracy of pose estimation than OpenPose ${ }^{3)}$.
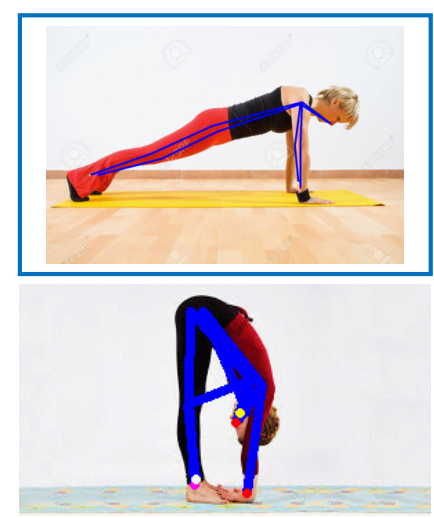

Fig. 7 Two pairs of estimated poses by the proposed method (left column) and OpenPose ${ }^{3)}$ (right column), where the better poses are marked with blue rectangles. It is usually challenging to estimate poses in these hard cases.

images for a hard menu. We use $80 \%$ of images as training data and the remaining $20 \%$ of images as test data. Note that we do not fine-tune OpenPose model using the collected dataset for fitness. Therefore, the higher accuracy may come from the effectiveness of more data, which infer that it is important to collect proper data to improve the accuracy of hard cases. In addition, note that the fine-tuned model is only used in fitness application.

In addition, to save network bandwidth, we limit the 


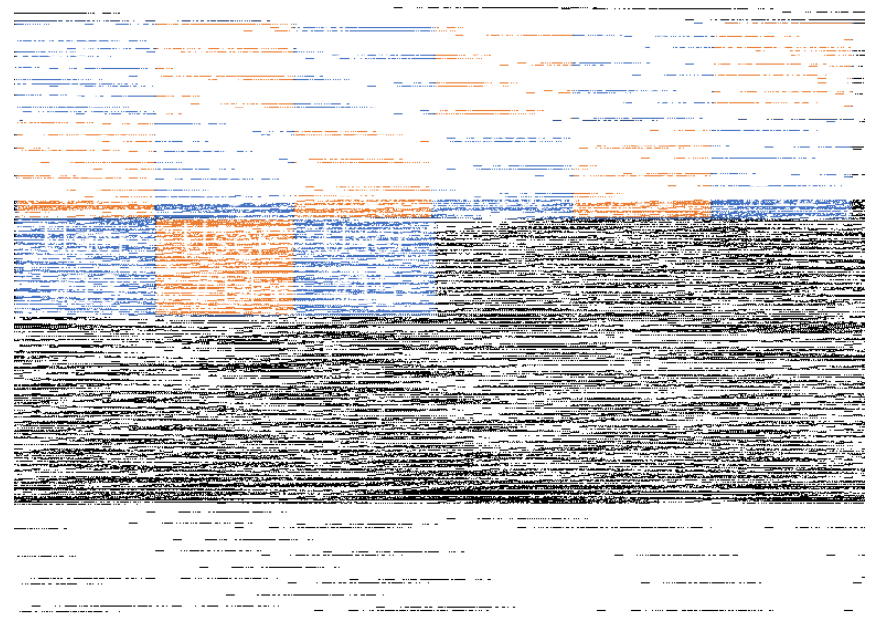

Fig. 8 Compose the videos from multiple users, where a maximum of 36 users can access just one GPU simultaneously.

resolution of videos that users send to the server. At the same time, we compose the videos from different users, as shown in Fig. 8, to further speed the computation required in the server. This method allows as many as 36 users to simultaneously access just one GPU, greatly reducing the cost in GPUs when providing the service to multiple users. If using Amazon EC2 G4 Instance (NVIDIA T4 GPU), the inference time is about $160 \mathrm{~ms}$ per frame. For each user, the processing speed is about $6.25 \mathrm{fps}$, which is fast enough for most actions in fitness service. In terms of throughput, the processing speed is about $225 \mathrm{fps}$ in the case of 36 users.

\section{Application to Speed Climbing}

Our system can provide many novel services of significant value in sports, aiming to support the training of athletes or create new experiences for viewing the sports $^{1125)}$. In this section, we will describe an application developed for speed climbing.

- Athlete training support: In July, 2020, KDDI constructed a climbing wall for TEAM au in Ibaraki Prefecture as shown in Fig. 9. The athletes in TEAM au use a smart-phone and a tablet to record the videos when they climb the wall, and then send the videos to our server via our application. The analysis results get sent back to their tablets. As shown in Fig. 10, the athletes can watch their own poses and trajectories during the climb, and thus be better able to understand their weak points and improve their skills more efficiently.

- New watching experience: During the contests, our system records $4 \mathrm{~K}$ videos with a $4 \mathrm{~K}$ consumer camera and generates new content with our analysis results in a short time, as shown in.Fig. 11 and Fig. 12

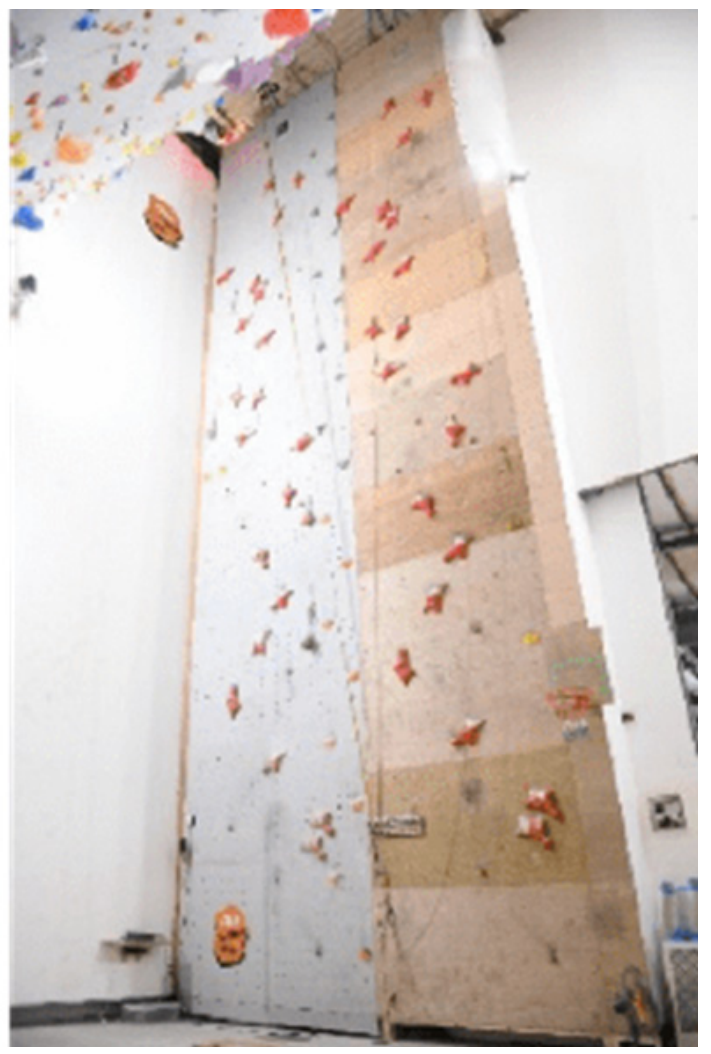

Fig. 9 A climbing wall was constructed by KDDI to support athletes in TEAM au. Together with the climbing wall, our application provides technical support for the athletes.

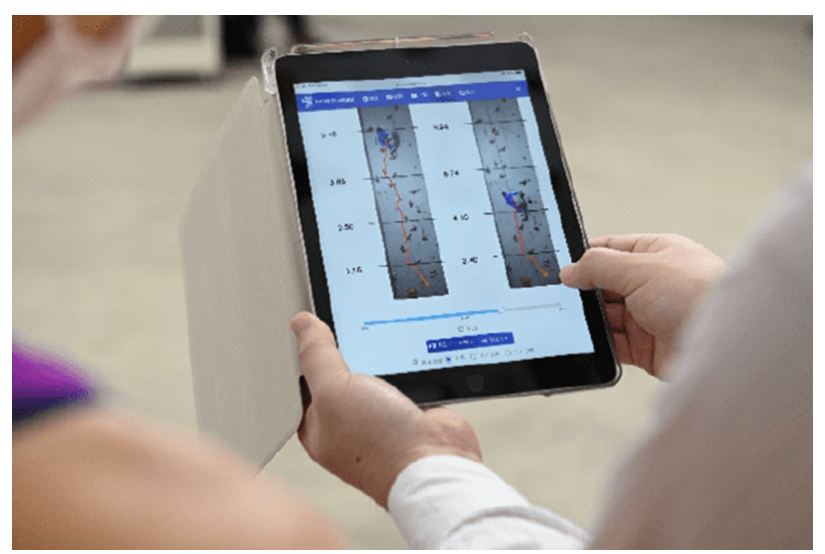

Fig. 10 Athletes are watching the analysis results during training, so they can check their weak points on a tablet.

(https://climbing-au.jp/post000141.html). Then, the generated content can be shown in a large display or broadcast live. The time difference from a world record is listed when the athletes pass certain key stones.

One technical issue in speed climbing is the shadow problem. Although the shadows are sometimes incorrectly detected as persons, it is easy to remove such errors in most cases. Because the confidence level of a shadow is usually much lower than that of a person, we select the highest confidence level as the output and 


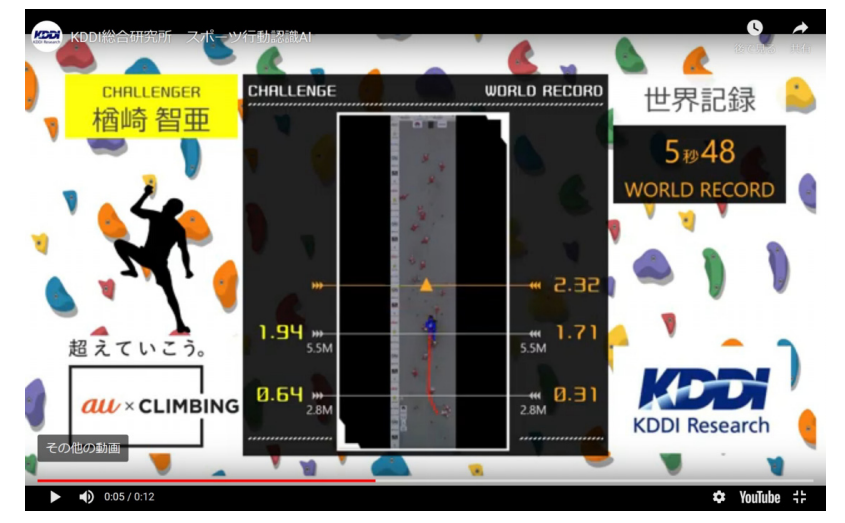

Fig. 11 Our system generates new content for speed climbing at au SPEED STARS 2019 SPEED CLIMBING CUP, which is displayed on a large monitor.

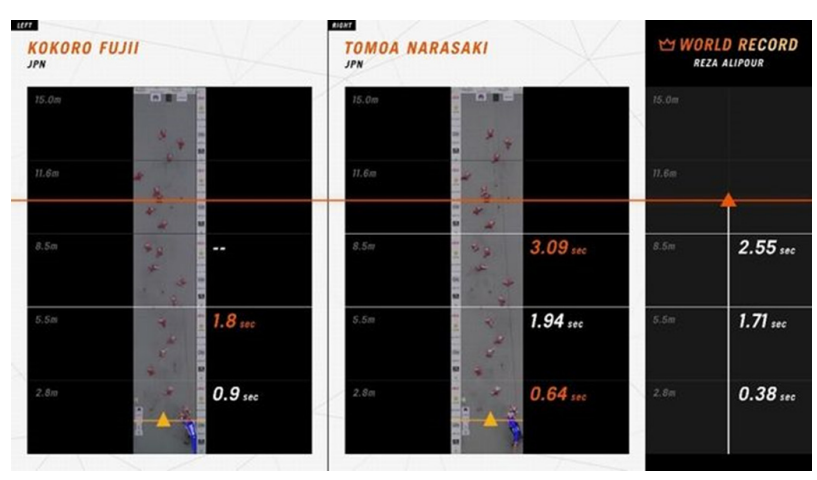

Fig. 12 Our system generates new content for speed climbing at IFSC Climbing World Cup 2019, which is broadcast live. Two athletes are shown simultaneously, side by side.

omit all the other bounding boxes. Unfortunately, this does not ensure perfection. As shown in Fig. 13 (a) and (b), the confidence levels of shadows in some frames are even higher than those of actual persons, leading to incorrect pose detection.

The basic idea of our solution comes from the observation that the accuracy of pose estimation is actually not affected much by the size of the bounding box. We conducted an experiment on the COCO dataset $^{22)}$, where the detected bounding boxes of persons are enlarged by $x$ times. The accuracy is calculated by COCO's standard metrics Object Keypoint Similarity (OKS) based $\mathrm{mAP}^{26}$. OKS is calculated from the distance between predicted points and ground truth points normalized by the scale of the person, whose value is from 0 to 1 (perfect prediction). In this paper, AP@0.75 denotes average precision at $\mathrm{OKS}=0.75$. The result in Table 1 shows that we can enlarge the bounding boxes by a factor of 1.5 without excessively decreasing the accuracy of pose estimation. Therefore, we merge the bounding boxes where the confidence level

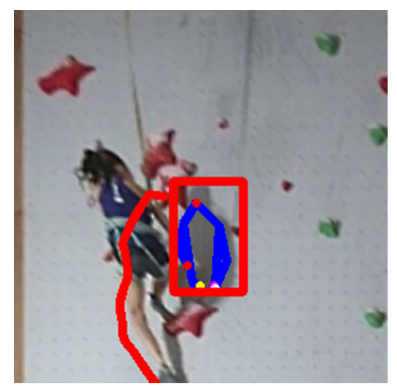

(a) Frame \#395

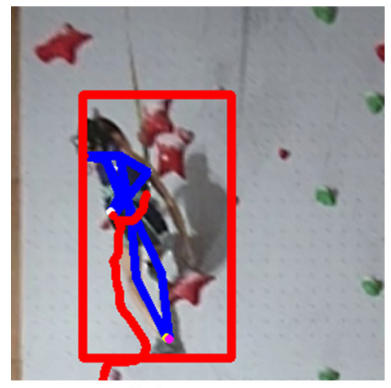

(c) Frame \#395

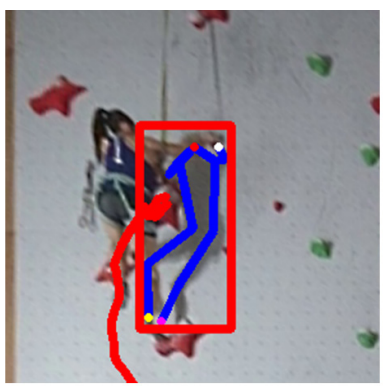

(b) Frame \#408

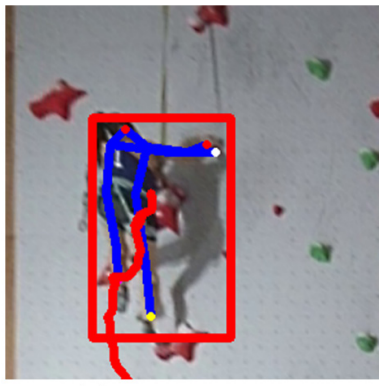

(d) Frame \#408
Fig. 13 Our solution to the shadow problem. Top: shadow area is incorrectly detected as person area. Bottom: person area is correctly detected although the detected area is a little larger.

Table 1 Accuracy comparison of pose estimation with the $\mathrm{COCO}$ dataset $^{22)}$ by using different size of bounding boxes. $x$ denotes the ratio to the original size of a bounding box.

\begin{tabular}{|c||c|c|c|}
\hline$x$ & AP@0.50:0.95 & AP@0.50 & AP@0.75 \\
\hline 1.0 & $69.7 \%$ & $88.3 \%$ & $77.0 \%$ \\
\hline 1.3 & $69.6 \%$ & $88.3 \%$ & $76.8 \%$ \\
\hline 1.5 & $68.2 \%$ & $87.9 \%$ & $76.2 \%$ \\
\hline
\end{tabular}

is higher than a threshold, which may include the person and the shadow as shown in Fig. 13 (c) and (d). As expected, the detected keypoints are still correct although the bounding box is large.

\section{Discussions on Multi-person Applica- tions}

In typical multi-person applications such as soccer, baseball, and basketball, there are several challenging issues on pose estimation. Given $4 \mathrm{~K}$ soccer videos as shown in Fig.14, we will discuss three of them, i.e., occlusion, small person areas, and heavy computing cost.

As shown in Fig.14, the areas of players are rather small (less than 100x100 pixels) in order to cover the field as much as possible. As shown in Fig. 15, it is rather difficult to estimate poses from a small person area. To achieve better accuracy of pose estimation, a deeper network model is preferred. However, there are more than 20 players in the field, which requires to use a lighter network model to make the processing faster. The trade-off between accuracy and computing speed 


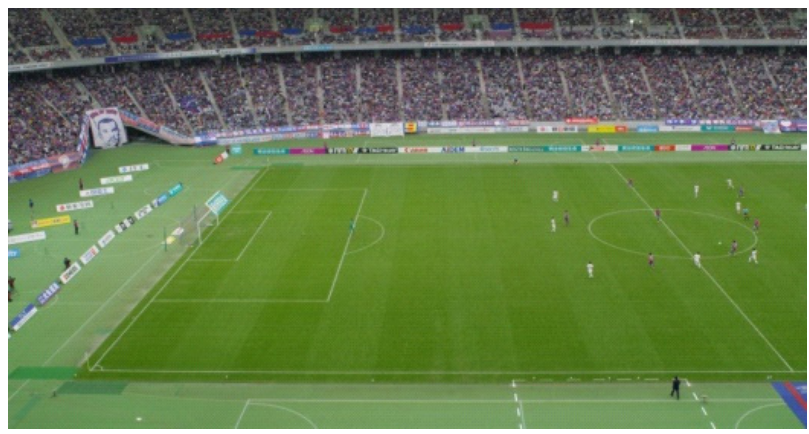

Fig. 14 A sample frame from $4 \mathrm{~K}$ soccer video, where multiple players are captured on the field.
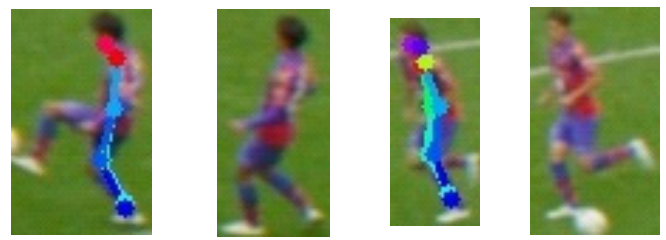

Fig. 15 It is rather difficult to estimate poses from a small person area.
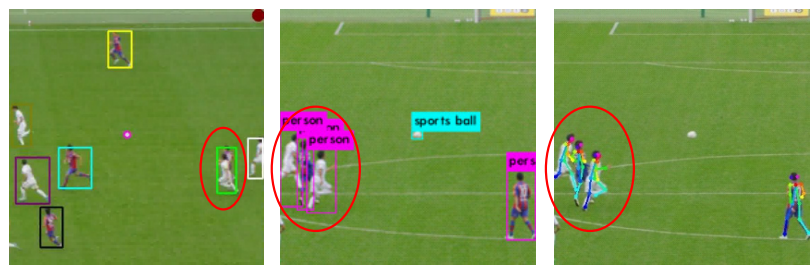

Fig. 16 Occlusion problem in soccer videos. Red ellipses show the missing person detections (left), correct person detections (middle), and missing pose detections (right)

is one of important issues in multi-person applications.

Another important issue is the occlusion problem as shown in Fig. 16. As a top-down approach, there are two types of failures due to the occlusion problem. One is that only a bounding box is detected from two persons as shown in the red ellipse of Fig. 16 (left). Another is that part of the occluded person is missing as shown in the red ellipse of Fig. 16 (right) although the bounding boxes are correctly detected as shown in the red ellipse of Fig. 16 (middle). One intuitive solution is to use multiple cameras from different capturing angles, which can avoid many occlusion cases but may still fail in a crowded scene. In addition, new networks such as ClueNet $^{27)}$ were proposed to solve the occlusion problem. Although it showed promising performance, the occlusion problem still remains unsolved due to lack of training data and many other issues.

\section{Conclusions}

In this paper, we presented a fast and accurate whole- body pose estimation system in the wild. Furthermore, we introduced two applications of our system: for fitness and speed climbing. In a real environment, we observed some technical issues and presented their solutions, which lead to better computing acceleration and detection accuracy.

\section{References}

1) J. Xu, K. Tasaka, and H. Yanagihara, "Beyond two-stream: Skeleton-based three-stream networks for action recognition in videos," in 2018 24th International Conference on Pattern Recognition (ICPR). IEEE, 2018, pp. 1567-1573.

2) Q. Dang, J. Yin, B. Wang, and W. Zheng, "Deep learning based 2d human pose estimation: A survey," Tsinghua Science and Technology, vol. 24, no. 6, pp. 663-676, 2019.

3) Z. Cao, T. Simon, S. E. Wei, and Y. Sheikh, "Realtime multiperson $2 \mathrm{~d}$ pose estimation using part affinity fields," in CVPR $2017,2017$.

4) Y. Chen, Z. Wang, Y. Peng, Z. Zhang, G. Yu, and J. Sun, "Cascaded Pyramid Network for Multi-Person Pose Estimation," 2018.

5) H. S. Fang, S. Xie, Y. W. Tai, and C. Lu, "RMPE: Regional multi-person pose estimation," in ICCV, 2017.

6) K. Sun, B. Xiao, D. Liu, and J. Wang, "Deep high-resolution representation learning for human pose estimation," in Proceedings of the IEEE conference on computer vision and pattern recognition, 2019, pp. 5693-5703.

7) S. Ren, K. He, R. Girshick, and J. Sun, "Faster r-cnn: Towards real-time object detection with region proposal networks," in Advances in neural information processing systems, 2015, pp. 91-99.

8) M. Fieraru, A. Khoreva, L. Pishchulin, and B. Schiele, "Learning to refine human pose estimation," in The IEEE Conference on Computer Vision and Pattern Recognition (CVPR) Workshops, June 2018.

9) J. Han, D. Zhang, G. Cheng, N. Liu, and D. Xu, "Advanced deep-learning techniques for salient and category-specific object detection: A survey," IEEE Signal Processing Magazine, vol. 35, no. 1, pp. 84-100, Jan 2018.

10) S. Jin, L. Xu, J. Xu, C. Wang, W. Liu, C. Qian, W. Ouyang, and P. Luo, "Whole-body human pose estimation in the wild," in European Conference on Computer Vision. Springer, 2020, pp. 196-214.

11) J. Xu, L. Kanokphan, and K. Tasaka, "Fast and accurate object detection using image cropping/resizing in multi-view $4 \mathrm{k}$ sports videos," in Proceedings of the 1st International Workshop on Multimedia Content Analysis in Sports, 2018, pp. 97-103.

12) Q. Dang, J. Yin, B. Wang, and W. Zheng, "Deep learning based 2d human pose estimation: A survey," Tsinghua Science and Technology, vol. 24, no. 6, pp. 663-676, 2019.

13) J. Redmon, S. Divvala, R. Girshick, and A. Farhadi, "You only look once: Unified, real-time object detection," in Proceedings of the IEEE conference on computer vision and pattern recognition, 2016, pp. 779-788.

14) J. Redmon and A. Farhadi, "Yolo9000: Better, faster, stronger," in 2017 IEEE Conference on Computer Vision and Pattern Recognition (CVPR), July 2017, pp. 6517-6525.

15) J. Redmon and A. Farhadi, "Yolov3: An incremental improvement," arXiv, 2018.

16) W. Liu, D. Anguelov, D. Erhan, C. Szegedy, S. Reed, C. Y. Fu, and A. C. Berg, "Ssd: Single shot multibox detector," in European conference on computer vision. Springer, 2016, pp. 21-37.

17) J. Huang, V. Rathod, C. Sun, M. Zhu, A. Korattikara, A. Fathi, I. Fischer, Z. Wojna, Y. Song, S. Guadarrama, et al., "Speed/accuracy trade-offs for modern convolutional object detectors," in IEEE CVPR, 2017.

18) Z. Cao, G. Hidalgo Martinez, T. Simon, S. Wei, and Y. A. Sheikh, "Openpose: Realtime multi-person $2 \mathrm{~d}$ pose estimation using part affinity fields," IEEE Transactions on Pattern Analysis and Machine Intelligence, 2019.

19) K. He, G. Gkioxari, P. Dollár, and R. Girshick, "Mask r-cnn," in Computer Vision (ICCV), 2017 IEEE International Conference on. IEEE, 2017, pp. 2980-2988.

20) K. Sun, B. Xiao, D. Liu, and J. Wang, "Deep high-resolution 
representation learning for human pose estimation," in $C V P R$, 2019.

21) F. Zhang, X. Zhu, H. Dai, M. Ye, and C. Zhu, "Distributionaware coordinate representation for human pose estimation," in Proceedings of the IEEE conference on computer vision and pattern recognition, 2020.

22) T. Y. Lin, M. Maire, S. Belongie, J. Hays, P. Perona, D. Ramanan, P. Dollár, and C. L. Zitnick, "Microsoft coco: Common objects in context," in European conference on computer vision. Springer, 2014, pp. 740-755.

23) Z. Cao, G. Hidalgo, T Simon, S. E. Wei, and Y. Sheikh, "OpenPose: realtime multi-person $2 \mathrm{D}$ pose estimation using Part Affinity Fields," in arXiv preprint arXiv:1812.08008, 2018.

24) T. Simon, H. Joo, I. Matthews, and Y. Sheikh, "Hand keypoint detection in single images using multiview bootstrapping," in Proceedings of the IEEE conference on Computer Vision and Pattern Recognition, 2017, pp. 1145-1153.

25) J. Xu and K. Tasaka, "Keep your eye on the ball: Detection of kicking motions in multi-view $4 \mathrm{k}$ soccer videos," ITE Transactions on Media Technology and Applications, vol. 8, no. 2, pp. 81-88, 2020.

26) B. Xiao, H. Wu, and Y. Wei, "Simple baselines for human pose estimation and tracking," in Proceedings of the European conference on computer vision (ECCV), 2018, pp. 466-481.

27) Perla Sai Raj Kishore, Sudip Das, Partha Sarathi Mukherjee, and Ujjwal Bhattacharya, "Cluenet: A deep framework for occluded pedestrian pose estimation.," in $B M V C, 2019$, p. 245.

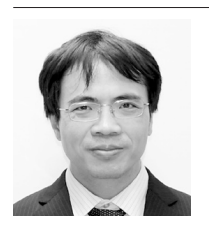

Jianfeng XU received B.S. (with honor) and M.S. degrees from Tsinghua University, China, in 2001 and 2004, and a Ph.D. from the University of Tokyo, Japan, in 2007. He has been working at KDDI Research, Inc. since 2007 and is now a research engineer in the Media Recognition Laboratory. His research interests include human motion analysis, sports analysis, and deep learning techniques.

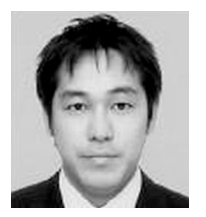

Kazuyuki TASAKA received M.S. and Ph.D. degrees from the Nara Institute of Science and Technology (NAIST), Japan, in 2004 and 2010. He has been working at KDDI Research, Inc. since 2004 and is now a group leader in the Media Recognition Laboratory. His research interests include human action recognition and dynamic map platforms.

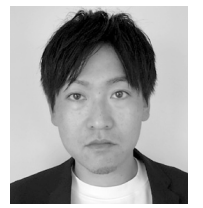

Masashi YAMAGUCHI received M.S. degree from Hosei University, Japan, in 2007. He has been working at KDDI Corporation since 2007 . After carrying out planning and development in product planning departments, he has engaged in overseas business launch and new business development for the $5 \mathrm{G}$ era in content service departments. 